\title{
INTEGRO-DIFFERENTIAL STOCHASTIC RESONANCE
}

\author{
LEVENTE TÖRÖK* and LÁSZLÓ B. KISH ${ }^{\dagger}$ \\ Texas A\&M University, Department of Electrical Engineering \\ College Station, Texas 77843-3128, USA \\ *torok@sztaki.hu, \\ †laszlo.kish@ee.tamu.edu \\ Received 15 September 2004 \\ Revised 1 February 2005 \\ Accepted 3 February 2005 \\ Communicated by Nigel Stocks
}

\begin{abstract}
A new class of stochastic resonator (SRT) and Stochastic Resonance (SR) phenomena are described. The new SRT consist of a classical SRT, one or more time derivative circuits and the same number of time integrators. The incoming signal with additive noise is first time derivated, then passes through the classical SRT and finally it is time integrated. The resulting SR phenomena show a well defined SR. Moreover the signal transfer and SNR are the best at the high frequency end. A particular property of the new system is the much smoother output signal due to the time integration.
\end{abstract}

Keywords: Smooth stochastic resonance; symmetric level crossing detector.

\section{Introduction}

Stochastic Resonators (SRTs) (see Refs. $[4,5,7]$ ) are one of the most intensively studied topic concerning noise assisted signal transfer. The particularity of these systems is that the signal power and signal-to-noise-ratio (SNR) at the output expresses well defined maxima versus the input noise strength.

Classical SRTs are threshold based devices. Therefore their output amplitude executes large abrupt jumps. This behavior is often disadvantageous in practical signal processing systems.

The motivation of the present work is to introduce a new class of SRTs and stochastic resonance (SR) phenomena which can produce arbitrary smooth amplitude while their SNR is as good as that of classical systems. Another important property of the new system that, under practical conditions, it has a frequencyresonance.

\footnotetext{
*Analogic and Neural Computing Systems Laboratory, Computer and Automation Research In-
} stitute of the Hungarian Academy of Sciences, H-1111, Budapest, Kende u. 13-17, Hungary. 
In the next sections, first the general integro differential stochastic resonator (IDSR) system is introduced in Sec. 2; then one appropriate realization we used is introduced in Sec. 3; the theoretical results are given in Sec. 4; and the simulation results are presented in Sec. 5 .

Finally, according to the fact, that we rely on the findings of Ref. [6] and it has not been published in journal, we survey the main results of this article with respect to the signal in the linear response limit.

\section{Integro-Differential Stochastic Resonators}

The integro-differential stochastic resonator (IDSRT) is a classical stochastic resonator with a time-derivation unit added before the output and a corresponding time-integration unit added at the output (see Fig. 1). Most of known stochastic resonators produce "rough" output containing "discontinuities", such as sharp spikes, random telegraph signals, abrupt switchings between amplitude levels. In an IDSRT, the expected "roughness" is significantly less because of the time-integration. Higher-order IDSRTs, with multiple derivators at the input and correspondingly multiple integrators at the output, will obviously produce an even smoother output. It is important to note that the smoothing effect works only on discontinuities added by the original SRT but not against discontinuities which may be found in the input signal.

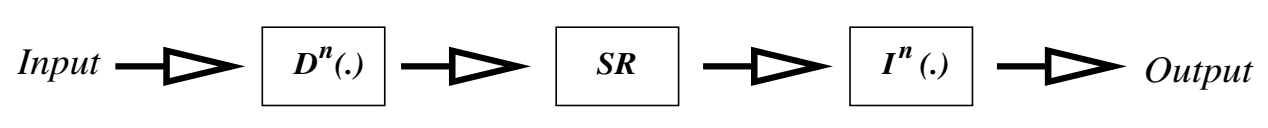

Fig. 1. Integro-Differential Stochastic Resonator system architecture.

\section{First Order Integro-Differential SRT with a Level-Crossing-Detector}

Having a well understood stochastic resonator, the so-called Level Crossing Detector (LCD), we applied it for the realization of the given model.

In general, an LCD emits spikes at its output whenever its input crosses a predefined threshold level. These spikes are now integrated in the new model, so instead of spikes stair like function appears at the output due to the integration. (see Fig. 2)

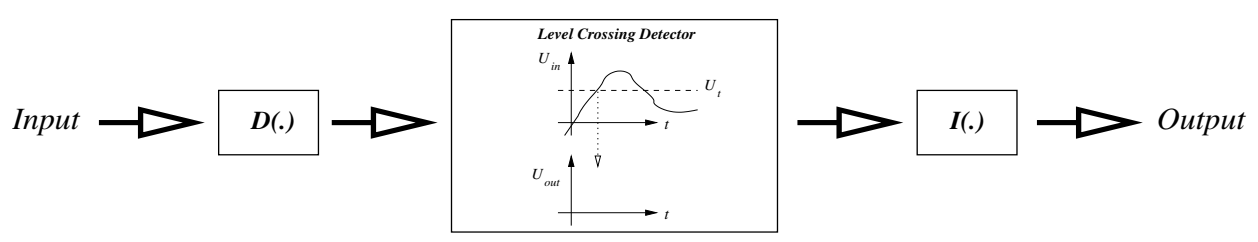

Fig. 2. Integro-Differential LCD architecture.

With higher order derivatives and higher order integrations we can get parabolic approximations which must be a "smooth" approach to the signal, particularly if it is sine wave function. 
One might think that applying a simple linear at the output of an SRT would produce a sufficiently smooth output. True, however the unpleasant phase shift would appear which does not happen if IDSRT is used.

On the other hand, we need to consider the fact that not only the signal is integrated in the post processing filter but the errors, too. In order to reduce its slowly varying component, not only the ideal integrator (i.e. numerical sum called stepwise integrator) but the leaky integrator was tested, as well.

In the next section we show how these particular linear input/output transformations affect the SR models in general.

\section{Theoretical Results}

In the processing line the derivator $\left(D^{n}()\right)$ of order of $(n)$ processes its input (denoted by in) providing the input for the LCD (denoted by diff). The LCD's output (denoted by $l c d$ ) is integrated $\left(I^{n}()\right)$ which is the output (denoted by int $=$ out) the entire process. (see Fig. 3).

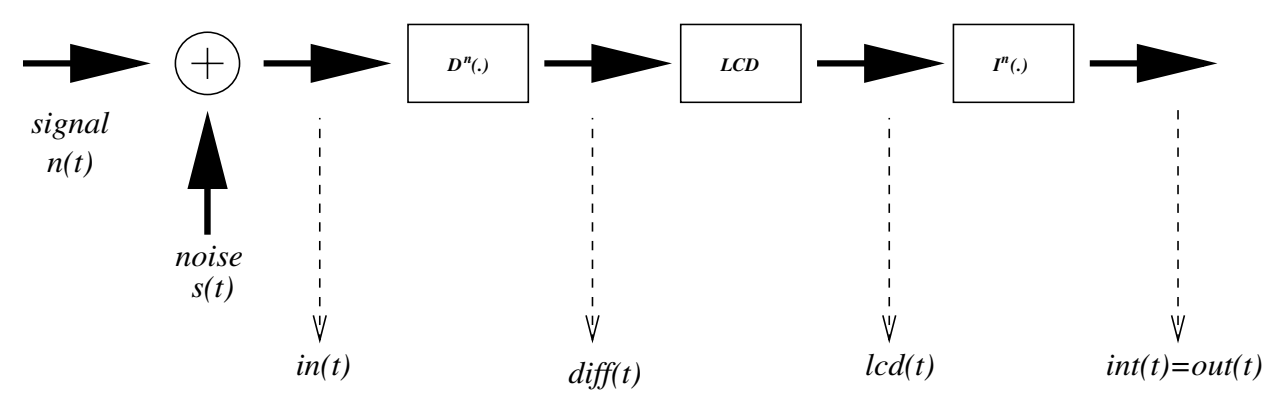

Fig. 3. Nomenclature of the measurement points of the system we will refer to. The symbol '+' in the circle is an adder.

The SNR is calculated for sinusoidal input singals as

$$
\operatorname{SNR}\left(f_{s}\right)=\frac{P}{S\left(f_{s}\right)},
$$

where $P$ is the signal contribution to the power spectral density (PSD) to the noise power spectra $(S(f))$ at $f_{s}$.

The main purpose of the theoretical analysis is to specify $\frac{S N R_{\text {out }}}{S N R_{\text {in }}}(f)$ analytically and derive its absolute maxima in explicit form.

\subsection{Input}

The input of the system (denoted by in $(t)$ ) consists of a hard limited white $(S(f)=S)$ noise in the frequency domain (denoted by $n(t)$ ) and a signal (denoted by $s(t))$;

$$
i n(t)=s(t)+n(t) .
$$

The cut-off frequency of band limited white noise is $f_{c}$ (i.e. the noise correlation time $f_{c} \approx 1 / \tau_{c}$ ). The rms of noise is denoted by $\sigma_{i n}$.

The signal is a sinusoidal wave with amplitude $A$ and frequency $f_{s}=\omega_{s} / 2 \pi$. 
The sinus wave signal's Fourier Transform (FT) can be written as $\hat{s}(f)=$ $A \delta\left(f_{s}-f\right)$, thus the power of such a signal is

$$
P_{\text {in }}=\frac{1}{2} \int_{0}^{\infty}(\hat{s}(f))^{2} d f=\frac{A^{2}}{2} .
$$

Since the white noise has uniform PSD, $S_{i n}=\frac{\sigma_{i n}^{2}}{f_{c}}$ holds. In this way,

$$
S N R_{i n}=\frac{A^{2} f_{c}}{2 \sigma_{i n}^{2}}
$$

\subsection{Derivation}

A derivation applied to $i n(t)$ does not change the $S N R(f)$ albeit changes the PSD of $P_{\text {diff }}\left(f_{s}\right)$ and $S_{\text {diff }}\left(f_{s}\right)$ compared to $P_{i n}$ and $S_{i n}$, respectively.

In general, a derivation in time domain (TD) is a multiplication by $\mathrm{j} \omega$ in the Fourier domain (FD)

$$
\hat{s}_{\text {diff }}(f)=\mathrm{j} 2 \pi f A \delta\left(f_{s}-f\right)=\mathrm{j} \omega A \delta\left(f_{s}-f\right) .
$$

For this reason, the power of the signal (or noise) becomes dependent on its frequency as

$$
P_{\text {diff }}\left(f_{s}\right)=\frac{1}{2} \int_{0}^{\infty}(\hat{s}(f))^{2} d f=\frac{1}{2} \int_{0}^{\infty}\left(\omega A \delta\left(f-f_{s}\right)\right)^{2} d f=\omega_{s}^{2} \frac{A^{2}}{2}
$$

which applies to the PSD of the noise too as

$$
\begin{aligned}
& P_{\text {diff }}\left(f_{s}\right)=\omega_{s}^{2} P_{\text {in }}\left(f_{s}\right) \\
& S_{\text {diff }}\left(f_{s}\right)=\omega_{s}^{2} S_{\text {in }}\left(f_{s}\right) .
\end{aligned}
$$

For this reason, in their ratio $\omega_{s}^{2}$ always simplifies as

$$
S N R_{\text {diff }}\left(f_{s}\right)=\frac{P_{\text {diff }}\left(f_{s}\right)}{S_{\text {diff }}\left(f_{s}\right)}=S N R_{\text {in }}\left(f_{s}\right) .
$$

While, the $S N R$ is intact under this linear transformation, the rms of the noise changes as,

$$
\sigma_{\text {diff }}^{2}=\int_{0}^{f_{c}} S_{d i f f}(f) d f=\int_{0}^{f_{c}}(2 \pi f)^{2} S_{i n} d f=\frac{4 \pi^{2}}{3} f_{c}^{3} S_{i n}=\frac{4 \pi^{2}}{3} f_{c}^{2} \sigma_{i n}^{2} .
$$

Note that, the noise of $\operatorname{diff}(t)$ is not white.

\section{3. $L C D$}

Definition 1. An asymmetric level crossing detector (LCD) emits an impulse of amplitude $(B)$ for duration of $\tau_{0}$ (uptime) on its output $(y(t)=l c d(t))$, whenever its input $(x(t)=\operatorname{dif} f(t))$ crosses the threshold level $\left(U_{t}\right)$ in increasing direction at time $\left(t_{i}\right)$.

$$
y(t)=L C D_{a s y m}(x(t))=B \sum_{i} 1\left(t-t_{i}\right)-1\left(t-\left(t_{i}+\tau_{0}\right)\right),
$$


where

$$
\left.x\right|_{t_{i}^{-}} \leq U_{t} \quad \text { and }\left.\quad x\right|_{t_{i}^{+}} \geq U_{t} .
$$

Definition 2. A symmetric level crossing detector (LCD) emits a positive impulse or a negative impulse for duration of $\tau_{0}$ on its output $(y(t)=l c d(t))$, whenever its input $(x(t)=\operatorname{dif} f(t))$ crosses the positive threshold level $\left(U_{t}\right)$ in increasing direction or crosses the negative threshold level $\left(-U_{t}\right)$ in decreasing direction at time $\left(t_{i}\right)$, respectively.

$$
y(t)=L C D_{\text {sym }}(x(t))=L C D_{\text {asym }}(x(t))-L C D_{\text {asym }}(-x(t)) .
$$

The SNR gain of an LCD is detailed in Ref. [6] and a brief is given in Appendix A, regarding the case when SNR is within the linear response limit. From there we recall the results we rely in the calculation of SNR gain of IDSRT.

Unfortunately, in case of the IDSR, the LCD is fed by non-white noise, so the result cannot be applied from there as a one-to-one replacement.

The average mean firing rate $\left(\nu_{0}\right)$ of a one-directional zero-crossings can be written similar to Eq. A.1 as

$$
\nu_{0}=\frac{2}{\sigma_{\text {diff }}}\left(\int_{0}^{f_{c}} f^{2} S_{\text {diff }}(f) d f\right)^{\frac{1}{2}}
$$

Because of the variation in the spectral characteristics $\left(S_{\text {diff }}(f)=(2 \pi f)^{2} S_{i n}\right)$, the integral in Eq. 6 can be written as

$$
\int_{0}^{f_{c}} f^{2}(2 \pi f)^{2} S_{i n} d f=4 \pi^{2} \frac{f_{c}^{5}}{5} S_{i n}=4 \pi^{2} \frac{f_{c}^{4}}{5} \sigma_{i n}^{2},
$$

so Eq. 6 simplifies to

$$
\nu_{0}=\frac{2}{\frac{2 \pi}{\sqrt{3}} f_{c} \sigma_{i n}} 2 \pi \frac{f_{c}^{2}}{\sqrt{5}} \sigma_{i n}=\frac{2 \sqrt{3}}{\sqrt{5}} f_{c} .
$$

The $S N R_{l c d}$ is similar to Eq. A.6 up to a substitution of the signal's amplitude in the IDSR setup which is $\omega_{s} A$, so it gets

$$
S N R_{l c d}=\frac{U_{t}^{2}}{\sigma_{i n}^{4}} \nu\left(U_{t}\right) \omega_{s}^{2} A^{2}
$$

Since Eq. 5 holds, by means of Eq. 2 the SNR gain $(\mu)$ is,

$$
\mu=\frac{S N R_{l c d}}{S N R_{d i f f}}=\frac{\frac{U_{t}^{2}}{\sigma_{i n}^{4}} \exp \left\{\frac{-U_{t}^{2}}{2 \sigma_{i n}^{2}}\right\} \frac{2 \sqrt{3}}{\sqrt{5}} f_{c} A^{2} \omega_{s}^{2}}{\frac{A^{2} f_{c}}{2 \sigma_{i n}^{2}}}=\frac{4 \sqrt{3}}{\sqrt{5}} \frac{U_{t}^{2}}{\sigma_{i n}^{2}} \exp \left\{\frac{-U_{t}^{2}}{2 \sigma_{i n}^{2}}\right\} \omega_{s}^{2} .
$$

Assuming $\alpha=\frac{U_{t}^{2}}{2 \sigma_{i n}^{2}}$ it simplifies to

$$
\mu=\frac{8 \sqrt{3}}{\sqrt{5}} \alpha \exp \{-\alpha\} \omega_{s}^{2} .
$$


The extrema of Eq. 7 is at $d \mu / d \alpha=0$ which results in $\left|\alpha_{\text {ext }}\right|=1$.

Using the considerations given in Appendix B regarding the symmetric LCD case opposed to the asymmetric, the maxima can be written as

$$
\mu=\frac{8 z \sqrt{3}}{\sqrt{5} \mathbf{e}} \omega_{s}^{2}
$$

where $z$ is 1 if asymmetric, or 2 if symmetric LCD is examined. One can conclude that the maxima of the system became signal frequency dependent.

\subsection{Integration}

Considerations similar to Sec. 4.2 apply to the integration. The only difference is in Eq. 3, where instead of multiplying by $\omega_{s}$, division must take place. Just as in Eq. 5, $\omega_{s}$ it will cancel. This means that by integrating the output of the LCD $(l c d(t))$, no SNR variation will appear;

$$
S N R_{\text {out }}=S N R_{l c d}
$$

so theoretically the Eq. 8 characterizes the overall performance.

It seems to be very attractive from the point of SNR gain since it provisions $S N R_{\text {gain }}>1$. According to the experiments, we have found that the IDSR hardly reaches the performance of the LCD because the signal amplitude (at the input of the LCD) goes out of the linear response limit as the frequency increases, however in the low frequencies regime the theory fits well to the experiments.

Note that, after the input derivation, the sinusoidal signal amplitude will be proportional to the frequency. So, if we increase the signal frequency, the signal gets greater at the LCD. However the noise is constant. As it is well-known, the non-linear response limit is reached when the signal amplitude at the input of the SRT is greater than the RMS noise. Exactly that happens in this case. This is another important point which shows that not only the input noise is transformed but also the input signal by this operation. We have a new system here which has a new behavior which is radically different from the classical SRTs.

\section{Simulations}

\subsection{Input - Gaussian White Noise Generation}

Large number of uniformly distributed random variables added together according to central limit theorem, provides accurate Gaussian process.

The rms of the noise was measured in every experiment rather than scaled after measuring in single experiment as is common.

Since the analog LCD simulation in Ref. [6], the fill factor of the impulse at the LCD's output was less than $10 \%$ in terms of the noise correlation time constant, first we built the experiment similar to that.

In order to reproduce the circumstances of the analog experiment, we needed a noise source at least 10 times slower than the impulse we can generate in the system. Equivalently, the noise had to be filtered by sharp low pass filter of 10th the cut-off frequency of the frequency corresponding to spike's uptime. While in TD, stepwise(we refer to it as oversampling) and linear-interpolation was tested, in FD, linear 
Finite Impulse Response filters were tested. By means of these techniques, we have found that the measurements are non sensitive for this particular fill factor a up to $100 \%$ fill factor. However we need to point out that aliasing type of errors might appear in simulations that has to be circumvented in all cases. It can be done by buffers of size of least common multiples and/or filtering in FD. We used the fastest case when the noise correlation time constant is equal to the uptime of the LCD, which means that every item in the buffer corresponds to a random sample and the LCD emits an impulse for duration of one sample.

\subsection{SNR measurements}

SNR measurement is based on separate signal and noise estimation.

Because of the structure of the experiment is such that we are in possession of the signal's frequency at the input (hence at the output), we can obtain the power of this particular frequency at the output by a simple "read out" from the measured PSD.

The background noise estimation is not that obvious. The particularly interesting noise is in the vicinity of the signal in the PSD. We measured it by simply averaging the PSD around the signal of a given narrow region. Specifically, we used fixed number of $+/-5$ frequency samples excluding $+/-1$ samples around the signal. (NB: since DFT was used frequency resolution is predefined)

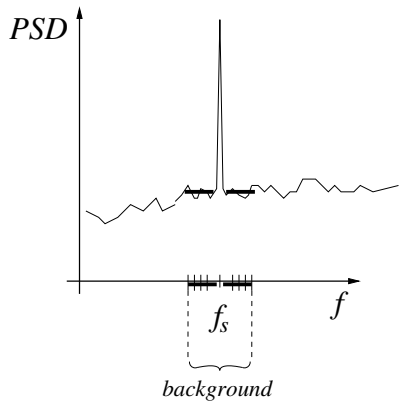

Fig. 4. The SNR measurement is based on the PSD. Signal's power can be determined by a simple "read out" at given frequency. The background noise is computed by averaging numerous samples of the PSD in the vicinity of the signal's frequency.

According to the findings of the Sec. $4, S N R$ s were checked on in, diff, lcd, out points of the system. The graphs generated by in this way are the base of our experimental analysis. (see Figs. 8 and 9).

\subsection{Derivation}

According to the numerical instability of the numerical derivation, the numerical curve considerably departs from the theoretical one in Fig. 7(b). Hence the Eq. 5 must hold up to the point of breakdown which implies that our measurement is supposed to be accurate no higher frequency than this. Thus Eq. 7 has at least the same high frequency limit. This can be justified in Fig. 5 where the $S N R_{\text {in }}$ and $S N R_{\text {diff }}$ versus signal frequency fits well in this region. 


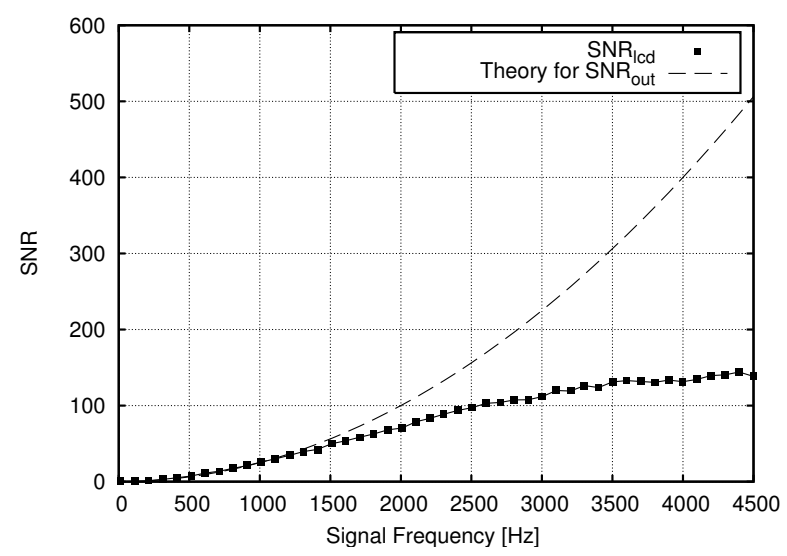

Fig. 5. SNR vs. signal frequency at different points in the system at a given rms. Theoretical $S N R_{\text {out }}$ curve is fitted to the numerical results in the numerically stable region. Theory explains signal transfer in the linear response limit which holds for up to $1500 \mathrm{~Hz}$.

\section{4. $L C D$}

Both asymmetrical and symmetrical LCD were tested with output impulse length equal to the noise sampling time. It is worth noticing that however the input noise of the LCD is colored, the output of the LCD is transformed to white noise in a considerable wide bandwidth which might have large potentialities in signal processing applications.

\subsection{Integration}

Achieving numerical integration in the TD leads to error prone results in the FD. Our experience supports this unreliability of the power spectra already show on Fig. 3.20 in Ref. [8]. We have found that this is due to the low representation degree of even conjugate signals with frequency lower than $1 / T$ in the FD obtained by FT (including DC component). Unfortunately the stepwise integration biases the spectra into this frequency range. In general, the integration formula widely used in FD, in case of non analytical functions performs very badly with Discrete FT (DFT). There at least two ways to avoid this numerical problem. Either windowing (e.g. Gaussian) or analog (or leaky) type of integration (with time constant $\tau \ll T / 2$ ) of the data in the DFT's buffer. We turned to leaky integration and tested the effect varying the integration time constant at $\tau=50 / f_{c}$ and $\tau=10 / f_{c}$.

\subsection{Experimental results}

In this section we give all the graphs of the measured systems. First, time series are shown, then PSDs are given, SNRs, SNR gain $\left(S N R_{\text {out }} / S N R_{\text {in }}\right)$, signal power versus signal frequency and versus rms.

A short time course of an experiment is demonstrated in Fig. 6.

Note the apparent positive tendency in Fig. 6(d) in the range of $0 . . .0 .05 s$ induced by the signal of $f_{s}=10 \mathrm{~Hz}$ which reflects the motivation of the IDSR setup, that is a smoother estimation of the signal at input by the output. 


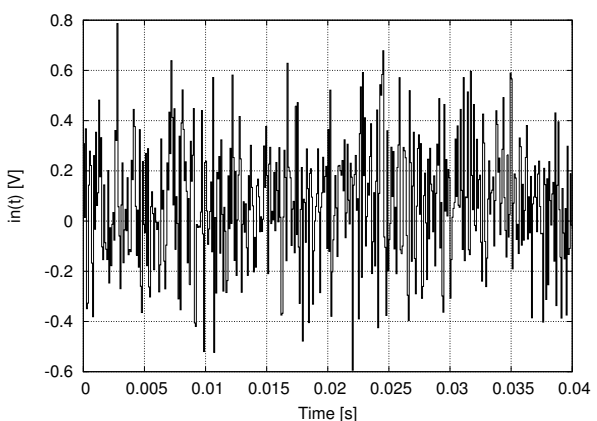

(a) The signal and the noise at the input.

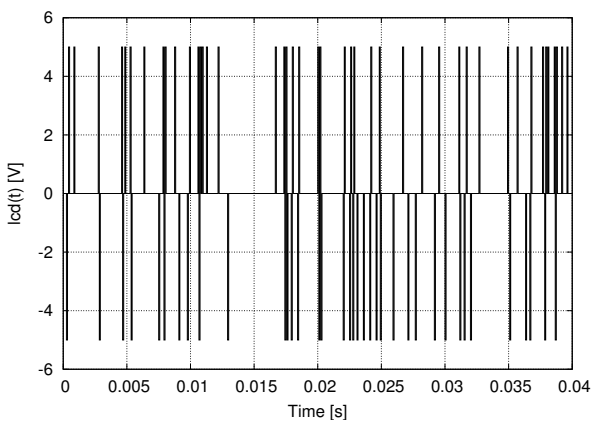

(c) The signal and the noise after the Stochastic Resonator realized as an LCD $(l c d)$.

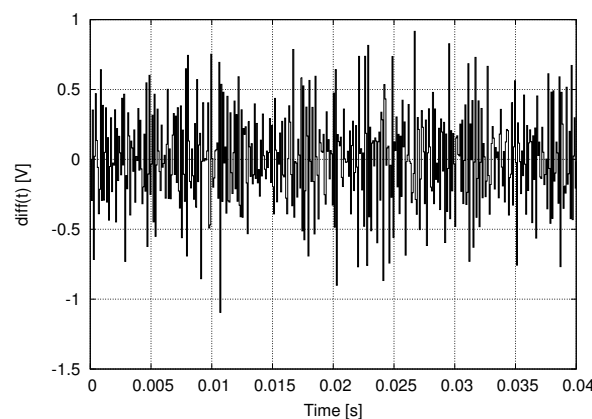

(b) The signal and the noise after derivation.

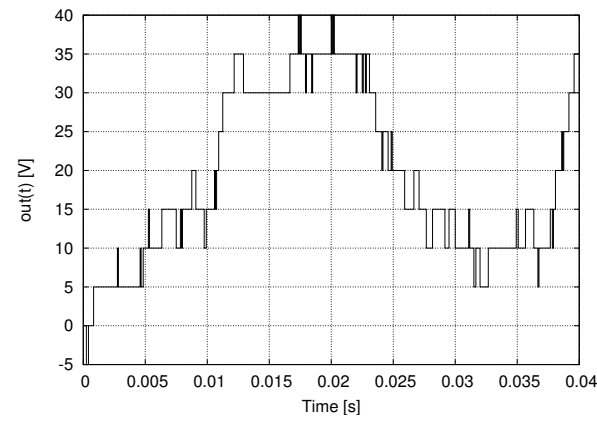

(d) Stepwise integration at the output of the LCD.

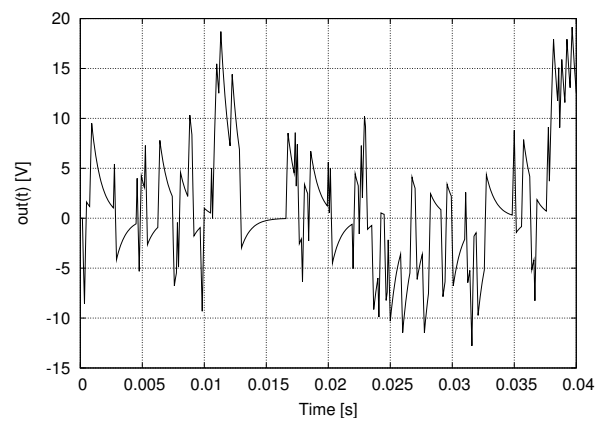

(e) Applying leaky integrator at the output of the LCD.

Fig. 6. Time course of noise+signal at different measurement points of the system within the same experimental setup. The applied configuration was: Symmetric LCD, $U_{t}=0.45, f_{c}=12000 \mathrm{~Hz}$, $A=0.1, B=5, \tau_{0}=0.833 \mu s, f_{s}=10 \mathrm{~Hz}$. 


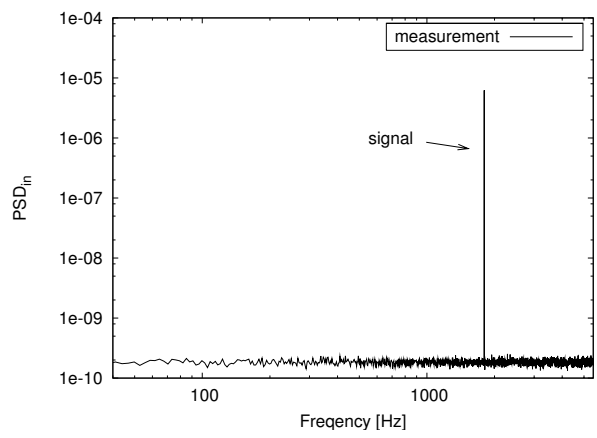

(a) The PSD of the input: white noise and single band signal.

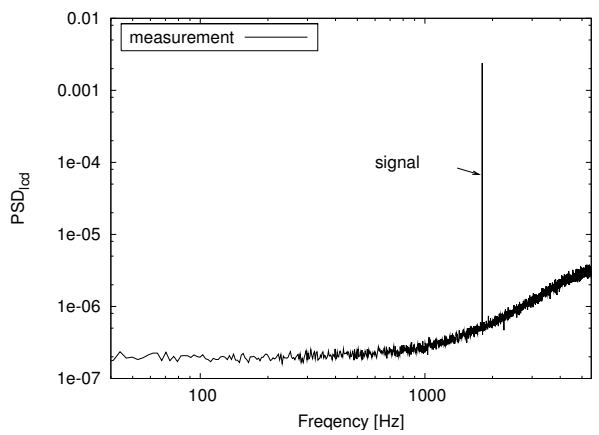

(c) The spectrum after a symmetric LCD Note that the frequency course is mostly white with the exception at the high end.

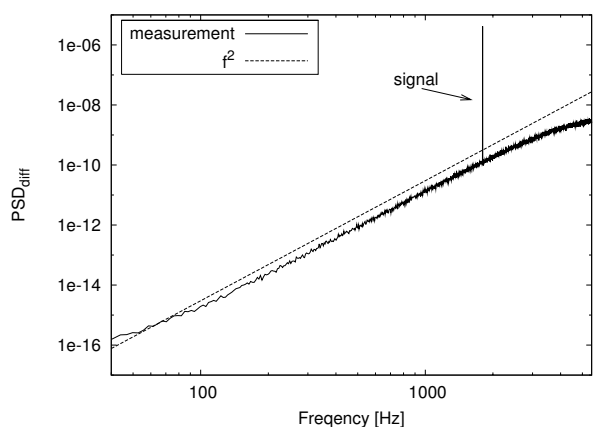

(b) The spectrum after derivation. Note the diversion from the ideal derivator which implies high frequency limit at about $4500 \mathrm{~Hz}$.

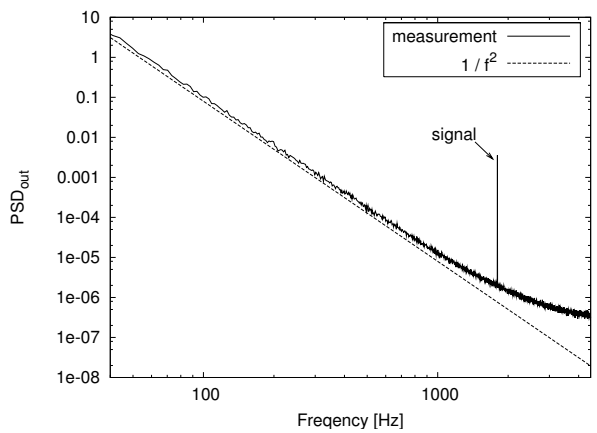

(d) The output of the system after integration.

Fig. 7. PSD of 500 averaged experiments with $U_{t}=0.45, f_{c}=12000 \mathrm{~Hz}, A=0.1, B=5$, $\tau_{0}=0.833 \mu \mathrm{s}, f_{s}=1800 \mathrm{~Hz}, \mathrm{rms}=0.2341$. The number of samples used was $N=4096$ that corresponds to $T=0.341 \mathrm{~s}$ time.

In Fig. 7, the PSD graphs of the same experiment is given in Fig. 6.

In the experiments, we used the following parametrization: Noise cut-off: $f_{c}=$ $12000 \mathrm{~Hz}$, signal amplitude: $A=0.1$, LCD threshold $U_{t}=0.45$, LCD impulse amplitude: $B=5$, LCD uptime: $\tau_{0}=0.833$. The scanned signal frequency range is $\{14 \mathrm{~Hz}, \ldots, 4500 \mathrm{~Hz}\}$ The rms was varied within the range of $\{0.01388 \ldots 0.5625\}$ in logarithmic steps (i.e. multiplied by 1.0559 in each step). Rms values were obtained by measurements rather than scaling the noise according to a single measurement.

In Fig. 8(a), SNRs are measured at different points in the system at a single rms using asymmetrical LCD with stepwise integrator. According to the expectations the $S N R_{i n}$ and $S N R_{\text {diff }}$ is equal for the whole regime tested. Note that the 


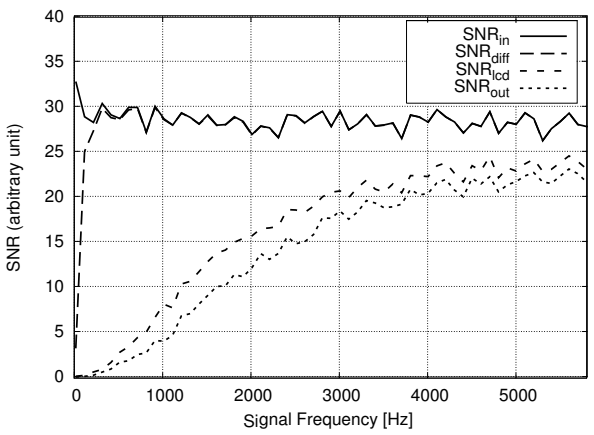

(a) The asymmetrical integro-differential system with stepwise integration. The maximal measured SNR gain is 0.827 .

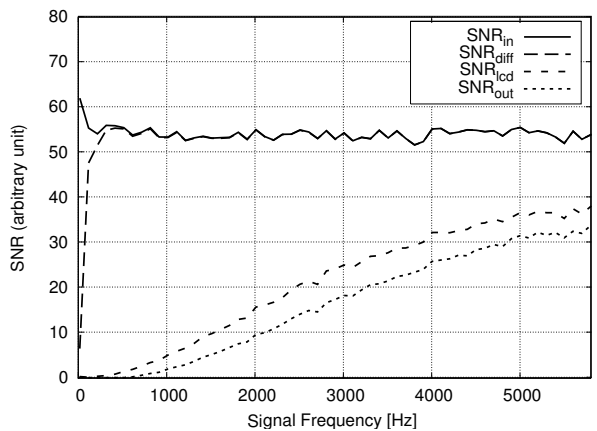

(c) The asymmetrical integro-differential system with leaky integrator $\left(\tau=50 / f_{c}\right)$. The maximal measured SNR gain is 0.626 .

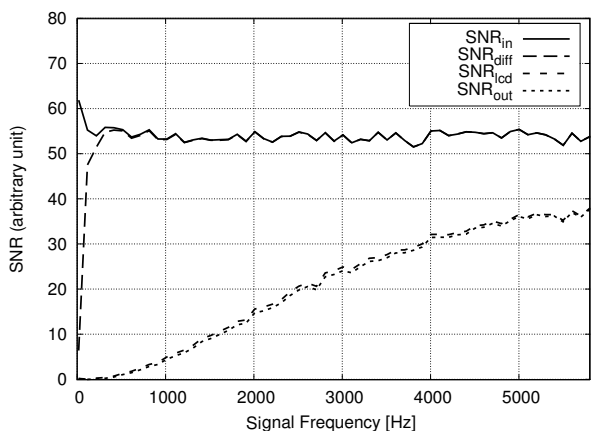

(e) The asymmetrical integro-differential system with leaky integration $\left(\tau=10 / f_{c}\right)$. The maximal measured SNR gain is 0.70 .

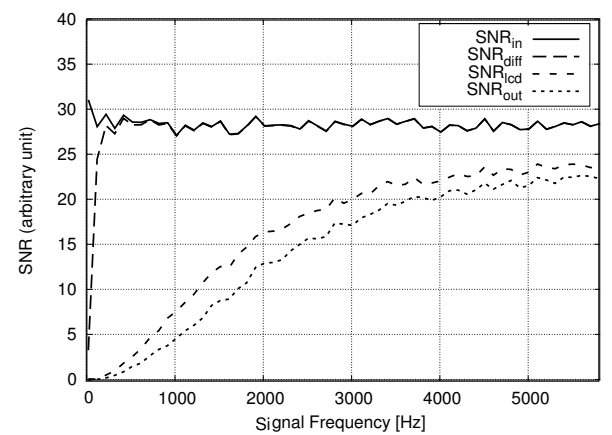

(b) The symmetrical integro-differential system with stepwise integration. The maximal measured SNR gain is 0.806 .

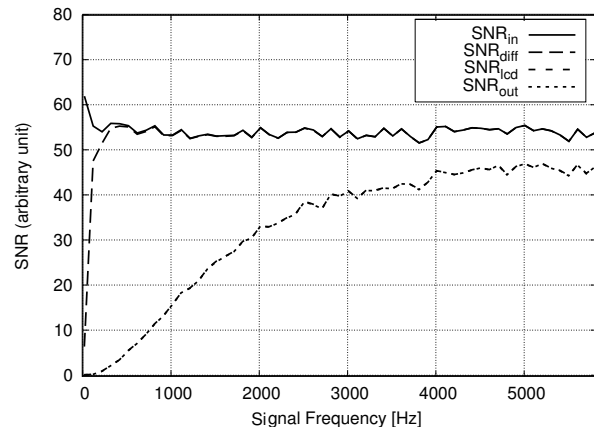

(d) $S N R_{\text {out }}$ versus signal frequency at a single rms (0.19). The symmetrical integro-differential system with leaky integration $\left(\tau=50 / f_{c}\right)$. The maximal measured SNR gain is 0.868 .

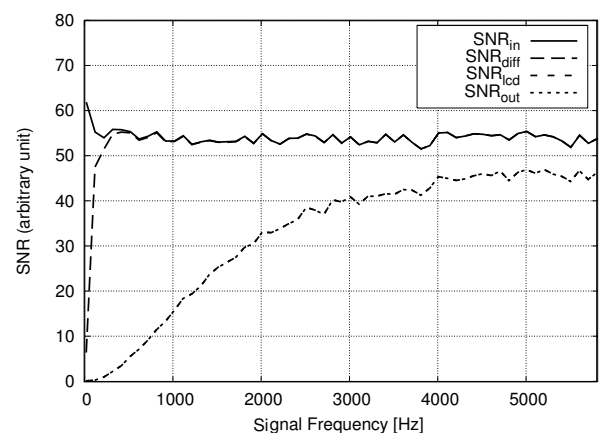

(f) The symmetrical integro-differential system with leaky integration $\left(\tau=10 / f_{c}\right)$. The maximal measured SNR gain is 0.866 .

Fig. 8. $S N R_{\text {out }}$ versus signal frequency at a single rms (0.19). The experimental setting was as in Fig. 7. 


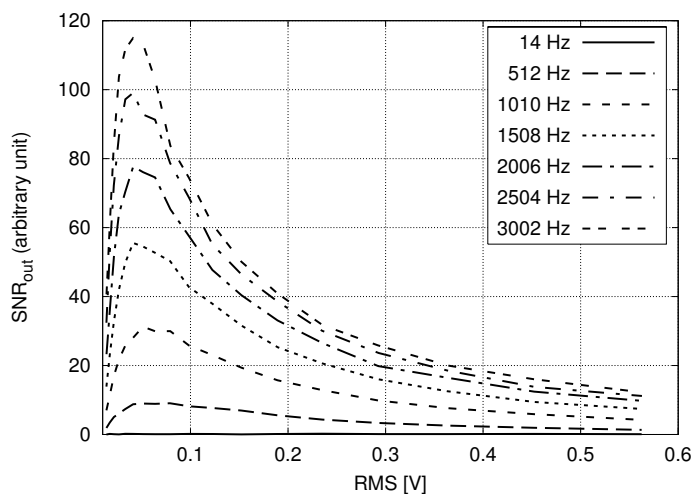

(a) $S N R_{\text {out }}$ versus rms at different signal frequencies.

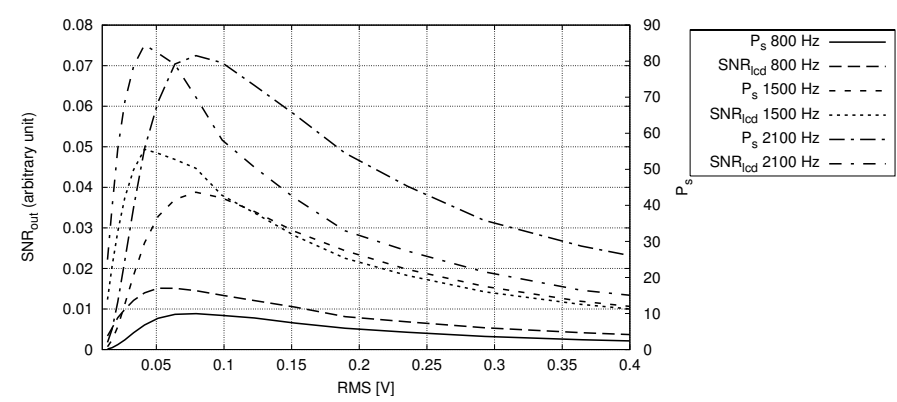

(b) $S N R_{\text {out }}$ and signal power versus rms at different signal frequencies.

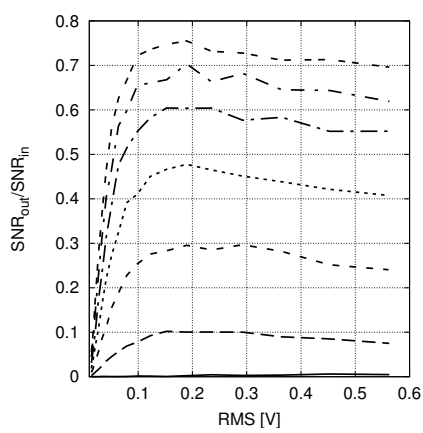

(c) $S N R_{\text {gain }}$ versus rms at different signal frequencies.

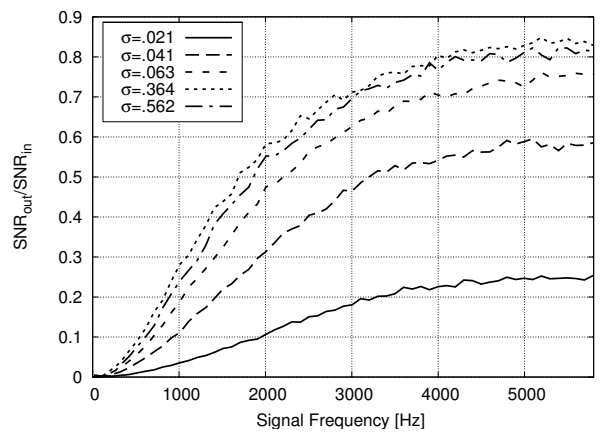

(d) $S N R_{\text {gain }}$ versus frequency at different rms's.

Fig. 9. The symmetrical integro-differential system with leaky integration $\left(\tau=10 / f_{c}\right)$. The experimental setting was as in Fig. 8. 
equivalence holds even over the breakdown point $(4500 \mathrm{~Hz})$ of the derivator. The output of the LCD fits well to the theoretical quadratic curve (just as in Fig. 5) but the output of the integrator departs qualitatively from this. The reason is already detailed in Sec. 5.5. As mentioned, the solution is twofold. Applying leaky integrator (see sub-figures $c, d, e, f$ in Fig. 8) (which reduces the low frequencies in the spectra) and symmetrical LCD (which reduces the DC component) (see subfigures $b, d, f$ in Fig. 8).

Interestingly in the experimental setup we used in generating all figures, the $S N R_{\text {out }}$ reaches its maxima at around $r m s=0.04$ at any signal frequency which would require further analysis. (see Fig. 9(a)).

It is demonstrated in Fig. 9(b) the maxima of the signal power and the maxima PSD at the same frequency is not at the same point as function of rms which is the proof for the noise assistance in the signal transfer.

Depending on the rms, the SNR gain increases rapidly in all experiments and reaches its maxima before 0.2 . By further increasing the rms the SNR gain remain constant (see in Fig. 9(c)) which means that the $S N R_{\text {out }}$ becomes proportional to the $S N R_{\text {in }}$ at almost around the maxima. This absolute maxima of all experiments goes just a little bit above (0.868) the well known LCD's limit (0.849) although it happens in the region where the derivation is declared to be unreliable. (see Fig. 9 and the Appendix)

\section{Conclusion}

We have introduced and demonstrated the viability of a new class of SRs. Another important characteristics of the system is signal and noise characteristics are the bests at the high frequency end, where most Shannon Information is transfered. (see Ref. $[2,9,10]$ )

\section{Acknowledgment}

The research was sponsored by the Office of Naval Research and the Army Research Office. LT's stay was partially financed by CARI-HAS Hungary. Thanks for Sergey M. Bezrukov for discussions.

\section{Appendix A.}

Here we brief the results of Kish from Ref. [6] with respect to a signal in the linear response limit of an LCD (see Def. 1. 2. ) fed by white noise and sinusoid signal.

The question of our interest is the maximal $S N R$ gain $\left(=S N R_{\text {in }} / S N R_{\text {out }}\right)$ that can be obtained from this system. $S N R_{\text {in }}$ can be formalized in a way as in Sec. 4.1 so Eq. 2 still holds.

To determine $S N R_{\text {out }}=S N R_{l c d}$, first, the mean impulse production frequency $\left(\nu\left(U_{t}\right)\right)$ is given as a function of constant input $s(t)=0$ with fixed threshold $U_{t}$ (see Rice formula in Ref. [1] for zero crossings and its generalization, for example, in Ref. [6] for non-zero threshold levels) as

$$
\nu\left(U_{t}\right)=\frac{2}{\sigma}\left(\int_{0}^{f_{c}} f^{2} S_{i n}(f) d f\right)^{\frac{1}{2}} \exp \left\{\frac{-U_{t}^{2}}{2 \sigma_{i n}^{2}}\right\}
$$


adding a signal $s(t)$ to the noise is essentially the same as modulating the threshold $U_{t}$ as $U_{t}^{s}=U_{t}-s(t)$, hence

$$
\nu\left(U_{t}^{s}\right)=\underbrace{\frac{1}{\sigma_{i n}}\left(\int_{0}^{f_{c}} f^{2} S_{i n}(f) d f\right)^{\frac{1}{2}}}_{\nu_{0}} \cdot \exp \left\{\frac{-\left(U_{t}-s(t)\right)^{2}}{2 \sigma_{i n}^{2}}\right\} .
$$

As a part of the expression, $\nu_{0}$ denotes the mean uni-directional zero crossing frequency of the input noise.

Assuming a white PSD for the noise $\left(S_{i n}(f)=S_{i n}=\sigma_{i n}^{2} / f_{c}\right), \nu_{0}$ can be written as,

$$
\nu_{0}=\frac{1}{\sigma_{i n}}\left(S_{i n} \int_{0}^{f_{c}} f^{2} d f\right)^{\frac{1}{2}}=\frac{1}{\sigma_{i n}} \sqrt{S_{i n}} \sqrt{\frac{f_{c}^{3}}{3}}=\frac{f_{c}}{\sqrt{3}}
$$

and the Taylor expansion of Eq. A.2 around $U_{t}$ is

$$
\begin{aligned}
\nu\left(U_{t}^{s}\right) & =\left(1+\frac{-U_{t}}{\sigma_{\text {in }}^{2}} s(t)+\ldots+\text { h.o.t. }\right) \nu\left(U_{t}\right) \\
& \approx \nu\left(U_{t}\right)+\underbrace{\left(\frac{-U_{t}}{\sigma_{\text {in }}^{2}} \nu\left(U_{t}\right)\right)}_{\nu_{\text {gain }}\left(U_{t}\right)} s(t)=\nu\left(U_{t}\right)+\nu_{\text {gain }}\left(U_{t}\right) s(t)
\end{aligned}
$$

where h.o.t. refers to higher order terms. Note that, the contribution of the signal is merely the last term. Since the signal is very slow compared to the mean firing rate of the Poisson process. (i.e. $\left.f_{s} \ll \nu\right)$ ), the time average of the impulse sequence emerged by reason of the modulation can be approximated as

$$
U_{a v}=B \tau_{0} \nu_{\text {gain }}\left(U_{t}\right) s(t)
$$

A device like this can be interpreted as an impulse-width modulator where the modulation is a sinus wave, which has the power

$$
P_{l c d}=\left(B \tau_{0} \nu_{\text {gain }}\left(U_{t}\right) A\right)^{2}
$$

Not like this is the background noise, which is a steady Poisson process with $\nu\left(U_{t}\right)$ mean repetition rate. Campbell's theorem (for $f>0$ ) applies to this as

$$
S_{l c d}(f)=\nu\left(U_{t}\right) B^{2} \frac{\left(\sin \left(\omega \tau_{0}\right)\right)^{2}}{\omega^{2}} .
$$

In the low frequency range, $\sin (x) / x \rightarrow 1$ so

$$
S_{l c d}=\nu\left(U_{t}\right) B^{2} \tau_{0}^{2}
$$

which leads to

$$
S N R_{l c d}=\frac{\nu_{g a i n}\left(U_{t}\right)^{2}}{\nu\left(U_{t}\right)} A^{2}
$$


By substituting $\nu_{\text {gain }}$,

$$
S N R_{l c d}=\frac{\frac{U_{t}^{2}}{\sigma_{i n}^{4}} \nu\left(U_{t}\right)^{2} A^{2}}{\nu\left(U_{t}\right)}=\frac{U_{t}^{2}}{\sigma_{i n}^{4}} \nu\left(U_{t}\right) A^{2} .
$$

Finally with Eq. 2 in the denominator, the SNR gain $(\mu)$ is

$$
\mu=\frac{S N R_{l c d}}{S N R_{i n}}=\frac{\left(U_{t}^{2} / \sigma_{i n}^{4}\right) \nu\left(U_{t}\right) A^{2}}{A^{2} f_{c} /\left(2 \sigma_{i n}^{2}\right)}=\frac{2}{f_{c}} \frac{U_{t}^{2}}{\sigma_{i n}^{2}} \nu\left(U_{t}\right) .
$$

By substituting Eq. A.1 (i.e. $\left.\nu\left(U_{t}\right)\right)$ in this by means of Eq. A.3,

$$
\mu=\frac{2}{f_{c}} \frac{U_{t}^{2}}{\sigma_{i n}^{2}} \frac{f_{c}}{\sqrt{3}} \exp \left\{-\frac{U_{t}^{2}}{2 \sigma_{i n}^{2}}\right\}=\frac{2}{\sqrt{3}} \frac{U_{t}^{2}}{\sigma_{i n}^{2}} \exp \left\{-\frac{U_{t}^{2}}{2 \sigma_{i n}^{2}}\right\} .
$$

This means that the SNR gain is independent of the applied signal frequency. Indeed, it seems to be independent of signal amplitude, too, but this is only due to linearization we made in Eq. A.4, so it is dependent but only up to the higher order terms effect.

To get the maxima consider using $\alpha=\frac{U_{t}^{2}}{2 \sigma_{i n}^{2}}$ so

$$
\mu=\frac{1}{\sqrt{3}} \alpha \exp \{-\alpha\}
$$

from which it can be seen that $d \mu / d \alpha=0 \rightarrow\left|\alpha_{\text {ext }}\right|=1$, hence the maxima is

$$
\max \left\{\frac{S N R_{l c d}}{S N R_{i n}}\right\}=\frac{4}{\mathbf{e} \sqrt{3}} .
$$

\section{Appendix B.}

Symmetric LCD's SNR can be concluded by assuming two asymmetric LCDs with $U_{t}^{+}=U_{t}$ and $U_{t}^{-}=-U_{t}$ in Eq. A.3, so the positive $\left(\nu^{+}\right)$and negative threshold crossing rates $\left(\nu^{-}\right)$are,

$$
\begin{aligned}
& \nu^{+}\left(U_{t}^{s}\right)=\nu_{0} \cdot \exp \left\{\frac{-\left(U_{t}-s(t)\right)^{2}}{2 \sigma_{i n}^{2}}\right\} \\
& \nu^{-}\left(U_{t}^{s}\right)=\nu_{0} \cdot \exp \left\{\frac{-\left(-U_{t}-s(t)\right)^{2}}{2 \sigma_{i n}^{2}}\right\} .
\end{aligned}
$$

After linearization as in Eq. A.4,

$$
\begin{aligned}
& \nu^{+}\left(U_{t}\right)=\nu\left(U_{t}\right)+\nu_{\text {gain }}\left(U_{t}\right) s(t) \\
& \nu^{-}\left(U_{t}\right)=\nu\left(U_{t}\right)-\nu_{\text {gain }}\left(U_{t}\right) s(t) .
\end{aligned}
$$

Since the positive and negative impulses,

$$
\begin{aligned}
& U_{a v}^{+}=B \tau_{0} \nu_{\text {gain }}\left(U_{t}\right) s(t) \\
& U_{a v}^{-}=(-B) \tau_{0} \nu_{\text {gain }}\left(U_{t}\right)(-s(t))
\end{aligned}
$$


are summed in the time domain, the average value

$$
U_{a v}^{s y m}=U_{a v}^{+}+U_{a v}^{-}=2 U_{a v}
$$

in terms of Eq. A.5, which means the signal power is $P_{l c d}^{s y m}=4 P_{l c d}$, while the background noise power is doubled only (i.e. $S_{l c d}^{s y m}=2 S_{l c d}$ ) since the two independent Poisson processes' power can be added. Thus their ratio, the SNR, is doubled compared to the asymmetric case $\left(S N R_{l c d}^{s y m}=2 S N R_{l c d}\right)$ which means the SNR gain is doubled, as well. By Eq. A.8, the maximal SNR gain, can be written in general form as,

$$
\max \left\{\frac{S N R_{l c d}}{S N R_{i n}}\right\}=\frac{4 z}{\mathbf{e} \sqrt{3}}
$$

where $z$ is 1 in asymmetric and 2 in symmetric LCD case.

This result is important since the this is the first time, as far as we are concerned, to get real SNR gain in the small signal limit with sinusoidal signals.

\section{References}

[1] S. O. Rice, Bell. Systems Technological Journal 23 (1944) 282; 46 (1945) 46.

[2] C. E. Shannon, Communication in the presence of noise, Proc. IRE 37 (1949) 10-21.

[3] Ch. Heiden, Power spectrum of stochastic pulse sequences with correlation between the pulse parameters, Physical Review 188 (1) (1969) 318-326.

[4] R. Benzi, A. Sutera and A. Vulpiani, The mechanism of stochastic resonance, J. Phys: Math. Gen. 14 (1981) 453-457.

[5] K. Wiesenfeld and F. Moss, Stochastic resonance and the benefits of noise: from ice ages to crayfish and SQUIDs, Nature 373 (1995) 33-36.

[6] L. B. Kiss, Possible Breakthrough: Significant Improvement of Signal to Noise Ratio by Stochastic Resonance, Chaotic, Fractals and Nonlinear Signal Processing, eds. R. Katz, American Institute of Physics Press, Mystic, Connecticut, USA 375 (1996) 880-897.

[7] S. M. Bezrukov and I. Vodyanoy, Stochastic resonance in non-dynamical systems without response thresholds, Nature 385 (1997) 319-321.

[8] R. B. Randall, (Bruel \& Kjaer) J. T. Broch and C. G. Wahrman, Chap. 3.2, Detectors, Frequency Analysis (1987) 87-101.

[9] L. B. Kiss, G. P. Hammer and D. Abbott, Information transfer rate of neurons: stochastic resonance of Shannon's information channel capacity, Fluct. Noise. Lett. 1 (2001) 13-17.

[10] S. M. Bezrukov and L. B. Kish, How much power does neural signal propagation need? Smart Mater Struct (2002) 800-803. 